\begin{tabular}{c} 
International Journal of Engineering \& Technology, $5(x)(2018) x x x-x x x$ \\
International Journal of Engineering \& Technology \\
SPC \\
Website www.sciencepubco.com/index.php/IJET \\
Roi: \\
Research paper, Short communication, Review, Technical paper \\
\hline
\end{tabular}

\title{
The Discrete Time-Space SIR-SI Age-Structured Model for Leptospirosis
}

\author{
Nor Azah Samat ${ }^{1 *}$, Aznida Che Awang ${ }^{2}$ \\ 1,2 Department of Mathematics, Faculty of Science and Mathematics, \\ Universiti Pendidikan Sultan Idris, 35900 Tanjong Malim, Perak, Malaysia. \\ *Corresponding author E-mail: norazah@fsmt.upsi.edu.my
}

\begin{abstract}
The aim of this study is to introduce new discrete time-space SIR-SI age-structured disease transmission models for Leptospirosis disease. The term "age-structured" here refers to having two different age groups, which are children and adult. These models are important because it will later lead to a conclusion of whether children or adult have high risk of being infected by Leptospirosis disease. In this study, two types of SIR-SI age-structured models are introduced. These include models with and without the transition rate between age groups. The future application of these two models will be based on the availability of parameter value and Leptospirosis data.
\end{abstract}

Keywords: Age-structured Model; Discrete Time-Space Mode; Leptospirosis; SIR Model.

\section{Introduction}

The infectious disease modelling can be divided into two groups which are direct disease transmission and indirect disease transmission. Direct disease transmission may happen when there is a direct physical contact between a susceptible person and infected person via blood or body fluids. In contrast, an indirect disease transmission refers to scenario where there is no human-to-human contact but the contact may happen through a reservoir of contaminated surfaces or from vectors such as rats, pigs, or dogs.

There are some differences between models for direct transmission and indirect transmission in which each model for direct transmission refers to the human population only but for indirect transmission, the first part of the model's acronym refer to the human population and the second part of the model's acronym refers to the vector population. The transmission models discussed in the studies by [1-3] are examples of indirect disease transmission.

There are two types of models that are useful in the study of infectious disease which are deterministic and stochastic. However, this paper focuses only on the deterministic model. The deterministic model is the model that is developed by data and known phenomenon that occur as a result of the data which is operated without randomness. The deterministic model is also known as a compartmental model where it gives the basic supporting structure for the study of transport between different systems. Therefore, a deterministic model helps us to determine and describe the average on the population scale and it is suitable to be used for large population. This model can be examined using differential or difference equation. Differential equation focuses on the continuous cases as opposed to discrete cases and time interval. In contrast, difference equation explains the transition between the different disease compartments using discrete time steps. In this study, discrete time interval, and difference equation for Leptospirosis disease is the focus of analysis.

\section{Disease Transmission Model for Leptospi- rosis}

This section discusses the basic SIR-SI model for transmission of Leptospirosis originally introduced by [4]. Then, the SIR-SI model that consider the age structure with involving the transition rate from children to adult human (Model 1) is proposed. Next, the model is improvised without involving the transition rate from children to adult human (Model 2). These three models only focus on the terms of compartmental model and mathematical difference equations.

\subsection{The Basic Discrete Time-Space SIR-SI Model for Leptospirosis}

Figure 1 shows the basic compartmental SIR-SI Model introduced by [4] for transmission of leptospirosis. The compartmental model is represented by boxes and arrows to connect between compartments. The solid arrow refers to a movement between the compartments while the dotted arrow refers to control signal. For example, in Figure 1, the dotted arrow from infectious vector compartment indicates that the number of infectious rat controls the number of infectious human compartment. Furthermore, the "arrow in" refers to the individuals entering the compartment and contributes to the positive sign in the difference equation while the "arrow out" refers to individuals exiting from the compartment and contributes to the negative sign in the difference equation as shown in (1).

In this compartmental model, the human group is divided into three sub-groups which are Susceptible( $S)$, Infectious $(I)$ and Recovered $(R)$ while the rat is divided into two sub-groups which are Susceptible $(S)$ and Infectious $(I)$. For the cases of leptospirosis, susceptible human can become infective and then recover from infection. However, rats will never recover from the infection because they will be infected for the rest of their lifetime [5].

Copyright $\odot 2018$ Authors. This is an open access article distributed under the Creative Commons Attribution License, which permits unrestricted use, distribution, and reproduction in any medium, provided the original work is properly cited. 
In Figure 1, for study regions, and time periods, a superscript $(H)$ is assigned to each compartment to represents human population, superscript $(V)$ represents vector or rat populations. Here, all the compartments are in the time period $j$ and study region $i$. Therefore, $S^{(H)_{i, j}}$ represents the number of human populations who are susceptible to the disease, but are not yet infected at time $j . I^{(H)}{ }_{i, j}$ represents the number of infected human populations and considered as infectious and $R^{(H)}{ }_{i, j}$ represents the number of recovered human populations who have been infected and then removed from the possibility of being infected again. Finally $S^{(V)_{i, j}}$ represents the number of susceptible vector and $I^{(V)}{ }_{i, j}$ represents the number of infectious vector.

Furthermore, in Figure 1, $\mu_{H}$ and $\mu_{v}$ represents the birth rate and death rate of human populations and vector populations, respectively by assuming equal birth rate and death rate. Besides that, $N_{H}$ and $N_{V}$ represent the total human populations and the total vector populations. Moreover, $r$ represents the recovery rate of human, $\beta_{H}$ represents the transmission rate of Leptospirosis from vector to human populations and lastly $\beta_{V}$ represents the transmission rate of Leptospirosis between vector populations.

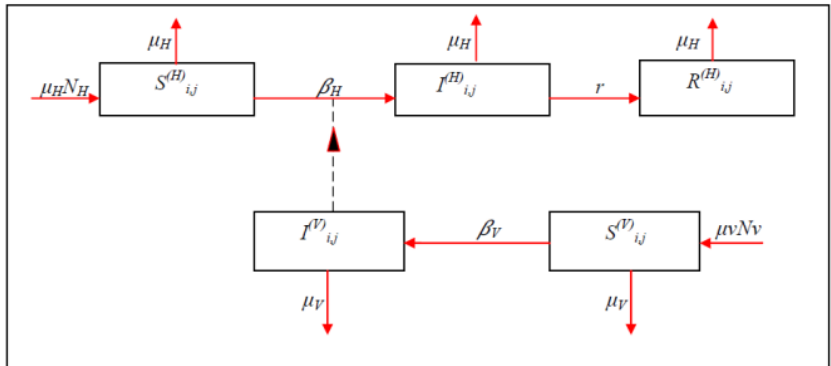

Fig. 1: The Basic Compartmental SIR-SI Model for the Transmission of Leptospirosis [4].

From Figure 1, it can be seen that the multiplication of birth rate of human and total human population will enter the susceptible human compartment, in which this compartment refers to the total number of person at risk. There will be some people dying due to natural death and some of the others may enter the infectious human compartment when they are infected by the leptospirosis disease from the infectious rats. At the infectious compartment, some people may die because of the natural death and some of them may recover from the disease and enter the recovery compartment. In that compartment, people may leave the compartment due to natural death.

Similar to the human compartmental process, vector populations is also subjected to the same process. In spite of this, the infectious population typically happens among the rats. However, there is no removal compartment for the rats because rat can become infective but they will not recover from infection.

The compartmental model shown in Figure 1 can be represented mathematically by a system of difference equations as follows:

For human population,

$S_{i, j}^{(H)}=\mu_{H} N_{H}+S^{(H)}{ }_{i, j-1}-\mu_{H} S^{(H)}{ }_{i, j-1}-\beta_{H} S^{(H)}{ }_{i, j-1} I^{(V)}{ }_{i, j-1}$

$I_{i, j}^{(H)}=\beta_{H} S_{i, j-1}^{(H)} I_{i, j-1}^{(V)}+I_{i, j-1}^{(H)}-\mu_{H} S_{i, j-1}^{(H)} I_{i, j-1}^{(V)}$

$R_{i, j}^{(H)}=r I_{i, j}^{(H)}+R_{i, j-1}^{(H)}-\mu_{H} R_{i, j-1}^{H}$

For vector population,

$S^{(V)}{ }_{i, j}=\mu_{V} N_{V}+S^{(V)}{ }_{i, j-1}-\beta \delta_{V} S^{(V)}{ }_{i, j-1} I^{(V)}{ }_{i, j-1}-\mu_{V} S^{(V)}{ }_{i, j-1}$

$I_{i, j}^{(V)}=\delta_{V} S_{i, j-1}^{(V)^{(V)}}{ }_{i, j-1}+I_{i, j-1}^{(V)_{1}}-\delta_{V} I_{i, j-1}^{(V)} I_{i, j-1}^{(V)}$
This study will then improve the basic SIR-SI model above by considering an age-structured variable in the new models. The improvement of the model is discussed in the next section.

\subsection{Model 1: The Discrete Time-Space SIR-SI Age- Structured Model}

This section discusses the compartmental model for transmission of leptospirosis in child and adult humans. According to [6], persons of all ages and gender are susceptible to be infected by leptospirosis but adult men are more susceptible because they tend to work in higher-risk jobs likes drainage cleaner, farm workers, campers, veterinarians and others. This is supported by statistic of leptospirosis cases during 2013 and 2014 that are provided by the Ministry of Health Malaysia in which the highest number of people infected by leptospirosis is the group of adult human.

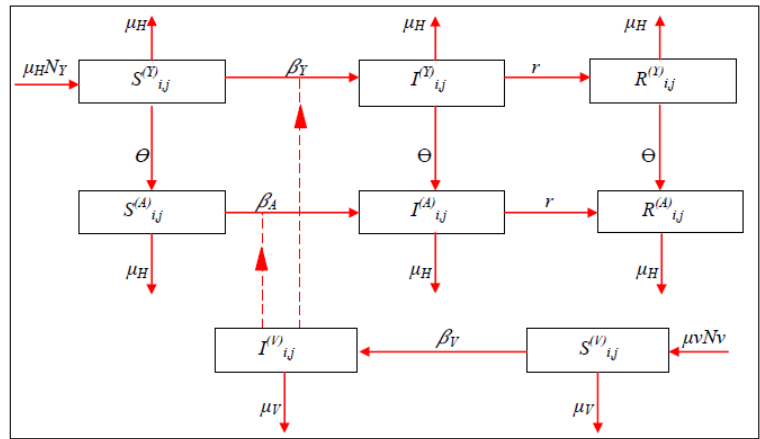

Fig. 2: The Compartmental SIR-SI Age-Structured Model for the Transmission of Leptospirosis

Figure 2 is the compartmental model that is adapted from [5] but has been improvised in terms of the notation as the earlier model does not consider the time and space. [5], take into account the transmission of leptospirosis disease between juvenile and adult humans in Thailand. However, human and vector populations have been separated into two parts of transmission diagram. This indicates that, the model did not consider the transmission rate between both populations. Conversely, in our study, the focus are on the transmission of leptospirosis disease between child and adult human as well as generating the compartment model for both populations in one diagram. Additionally, this study has classified children as people aged 0-17 years and adults as people aged 18 years and above. The classification of age for juvenile and adult in this study is based on Age of Majority Act 1971 in Law of Malaysia.

In Figure 2, for study regions, and time periods, a superscript $(Y)$ is assigned to each compartment to represent children human population, superscript $(A)$ represents adult human populations and superscript $(V)$ represents vector or rat populations. Here, all the compartments are in the time period $\mathrm{j}$ and study region $\mathrm{i}$. Therefore, $S^{(Y)} i, j$ represents the number of susceptible children human populations, $\left.I^{(Y)}\right)_{i, j}$ represents the number of infected children human populations and $R^{(Y)}{ }_{i, j}$ represents the recovered children human populations. While for adult human populations, $S^{(A)}{ }_{i, j}$ represents the number of susceptible adult, $I^{(A)}{ }_{i, j}$ represents the number of infectious adult and $R^{(A)}{ }_{i, j}$ represents the number of recovered adult. Finally $S^{(V)}{ }_{i, j}$ represents the number of susceptible vector and $\left.I^{(V)}\right)_{i, j}$ represents the number of infectious vector.

Furthermore, in Figure 2, $\theta$ represents the transition rate from children to adult human, $\beta Y$ represents the transmission rate of Leptospirosis from vector to children human populations, $\beta A$ represents the transmission rate of Leptospirosis from vector to adult human populations and lastly $\beta V$ represents the transmission rate of Leptospirosis from between vector populations. The definition of other notations in Figure 2 is similar as explained in previous section of basic SIR-SI model. 
The compartmental model shown Figure 2 can be represented mathematically by a system of difference equations as follows:

For children human population,

$$
\begin{aligned}
S^{(Y)}{ }_{i, j}= & \mu_{H} N_{H}+S^{(Y)_{i, j-1}}-\mu_{H} S^{(Y)_{i, j-1}}-\theta S^{(Y)}{ }_{i, j-1}- \\
& \beta_{Y} S^{(Y)}{ }_{i, j-1} I^{(V)}{ }_{i, j-1} \\
I^{(Y)}{ }_{i, j}= & \beta_{Y} S^{(Y)}{ }_{i, j-1} I^{(V)}{ }_{i, j-1}+I^{(Y)}{ }_{i, j-1}-\mu_{H} I^{(Y)}{ }_{i, j-1}- \\
& \theta I^{(Y)}{ }_{i, j-1}-r I^{(Y)}{ }_{i, j-1} \\
R^{(Y)}{ }_{i, j}= & r I^{(Y)}{ }_{i, j}+R^{(Y)_{i, j-1}}{ }_{i}-\mu_{H} R^{(Y)}{ }_{i, j-1}-\theta R^{(Y)}{ }_{i, j-1}
\end{aligned}
$$

For adult human population,

$$
\begin{aligned}
S^{(A)}{ }_{i, j}= & \theta S^{(Y)}{ }_{i, j}+S^{(A)}{ }_{i, j-1}-\beta_{A} S^{(A)}{ }_{i, j-1} I^{(V)}{ }_{i, j-1}- \\
& \mu_{H} S^{(A)}{ }_{i, j-1} \\
I^{(A)}{ }_{i, j}= & \beta_{A} S^{(A)}{ }_{i, j-1} I^{(V)}{ }_{i, j-1}+I^{(A)}{ }_{i, j-1}+\theta I^{(Y)}{ }_{i, j}+I^{(A)}{ }_{i, j-1} \\
& -r I^{(A)}{ }_{i, j-1}-\mu_{H} I^{(A)}{ }_{i, j-1}
\end{aligned}
$$$$
R_{i, j}^{(A)}=r I_{i, j}^{(A)_{i}}+\theta R_{i, j}^{(Y)}+R_{i, j-1}^{(A)}-\mu_{H} R_{i, j-1}^{(A)}
$$

For vector population,

$$
\begin{aligned}
& S^{(V)}{ }_{i, j}=\mu_{V} N_{V}+S^{(V)}{ }_{i, j-1}-\beta_{V} S^{(V)}{ }_{i, j-1} I^{(V)}{ }_{i, j-1}-\mu_{V} S^{(V)}{ }_{i, j-1} \\
& I^{(V)}{ }_{i, j}=\beta_{V} S^{(V)}{ }_{i, j-1} I^{(V)}{ }_{i, j-1}+I^{(V)}{ }_{i, j-1}-\mu_{V} I^{(V)}{ }_{i, j-1}
\end{aligned}
$$

Figure 2 shows the model which considers the transition rate from children to adult compartments. However, due to the unavailability of the data and time period of collected data restriction, this model is somehow not suitable for a short period analysis. This is because the transition from children to adult involves a very long time period. Since, it will take a year for an individual to move from one age group to another age group, so this model will be good for a long-term research because the researcher can follow up the condition of each patient.

Therefore, a new compartmental SIR-SI model for the transmission of leptospirosis disease in children and adult humans that omitted the transition rate from children to adult human is discussed in the next section.

\subsection{Model 2: The Discrete Time-Space Age-Structured SIR-SI Model}

Figures 3 and Figure 4 display the proposed compartmental models for leptospirosis disease in children and adult human, respectively. These compartmental models exclude the transition rate from children to adult. Therefore, children and adult in this model consider two separate age groups data at one period of time. The definitions of the notations used in the model are similar as explained in the previous section.

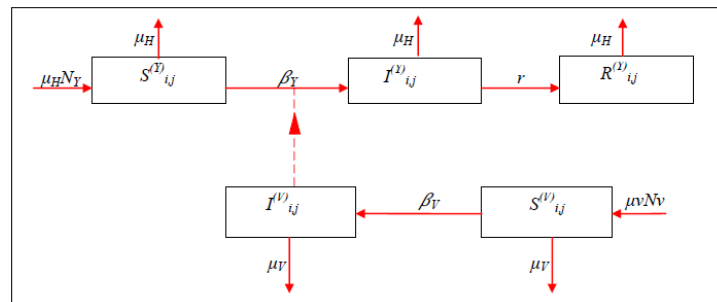

Fig. 3: The Compartmental SIR-SI Model for the Transmission of Leptospirosis in Children
Since this research consider the discrete time data, the compartmental model in Figure 3 can be expressed mathematically in the deterministic model given as follows:

For children human population,

$$
\begin{aligned}
& S^{(Y)}{ }_{i, j}=\mu_{H} N_{H}+S^{(Y)}{ }_{i, j-1}-\mu_{H} S^{(Y)}{ }_{i, j-1}-\beta_{Y} S^{(Y)}{ }_{i, j-1} I^{(V)}{ }_{i, j-1} \\
& \begin{aligned}
I_{i, j}^{(Y)}= & \beta_{Y} S^{(Y)}{ }_{i, j-1} I^{(V)}{ }_{i, j-1}+I^{(Y)}{ }_{i, j-1}-\mu_{H} I^{(Y)}{ }_{i, j-1}- \\
& r I^{(Y)}{ }_{i, j-1}
\end{aligned} \\
& R_{i, j}^{(Y)}=r I^{(Y)}{ }_{i, j}+R^{(Y)}{ }_{i, j-1}-\mu_{H} R^{(Y)}{ }_{i, j-1}
\end{aligned}
$$

For vector population,

$$
\begin{aligned}
& S^{(V)}{ }_{i, j}=\mu_{V} N_{V}+S^{(V)_{i, j-1}}-\beta_{V} S^{(V)}{ }_{i, j-1} I^{(V)}{ }_{i, j-1}-\mu_{V} S^{(V)}{ }_{i, j-1} \\
& I^{(V)}{ }_{i, j}=\beta_{V} S^{(V)}{ }_{i, j-1} I^{(V)}{ }_{i, j-1}+I^{(V)}{ }_{i, j-1}-\mu_{V} I^{(V)}{ }_{i, j-1}
\end{aligned}
$$

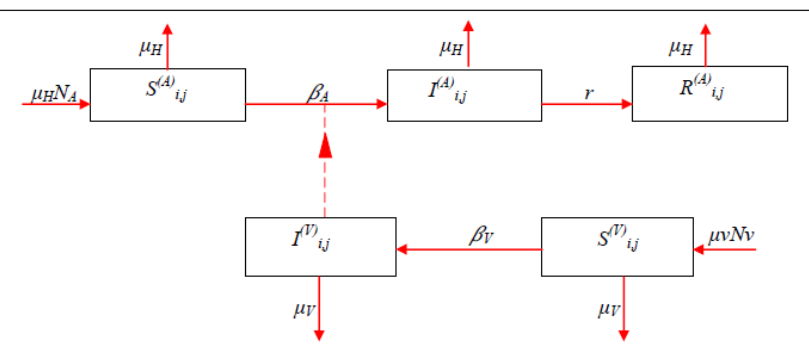

Fig. 4: The Compartmental SIR-SI Model for the Transmission of Leptospirosis in Adult

Next, the compartmental model shown in Figure 4 can be represented mathematically by a system of difference equations given as follows:

For adult human population,

$S_{i, j}^{(A)}=\mu_{H} N_{H}+S^{(A)}{ }_{i, j-1}-\mu_{H} S^{(A)}{ }_{i, j-1}-\beta_{A} S^{(A)}{ }_{i, j-1} I^{(V)}{ }_{i, j-1}$

$I_{i, j}^{(A)}=\beta_{A} S^{(A)}{ }_{i, j-1} I^{(V)}{ }_{i, j-1}+I^{(A)}{ }_{i, j-1}-\mu_{H} I_{i, j-1}^{(A)}-$ $r I^{(A)}{ }_{i, j-1}$

$R_{i, j}^{(A)}=r I_{i, j}^{(A)}+R_{i, j-1}^{(A)}-\mu_{H} R_{i, j-1}^{(A)}$

For vector population,

$S^{(V)}{ }_{i, j}=\mu_{V} N_{V}+S^{(V)}{ }_{i, j-1}-\beta_{V} S^{(V)}{ }_{i, j-1} I^{(V)}{ }_{i, j-1}-\mu_{V} S^{(V)}{ }_{i, j-1}$

$I^{(V)}{ }_{i, j}=\beta_{V} S^{(V)}{ }_{i, j-1} I^{(V)}{ }_{i, j-1}+I^{(V)_{i, j-1}}-\mu_{V} I^{(V)}{ }_{i, j-1}$

Model 2 is expected can be directly apply in the analysis of closed population data of SIR-SI model, in which the data collected is naturally in the form of two age groups data.

\section{Conclusion and Future Works}

A mathematical model is developed in order to understand the real life situation specifically for disease transmission. In this study, the compartmental and deterministic age-structured SIR-SI models are introduced to explain how a person is mutually exclusively moved from one compartment to another in one moment in time. The models introduced in this study can be used by the authorities 
or researchers as an investigative tool in understanding and reducing the Leptospirosis cases, especially cases according to age groups. Future works of this study include, improving the deterministic model into stochastic model and applying it to estimate the relative risk for Leptospirosis

\section{References}

[1] Samat NA \& Percy DF, Vector-borne infectious disease mapping with stochastic difference equations: an analysis of dengue disease in Malaysia, Journal of Applied Statistics, 39(9), (2012), pp.20292046.

[2] Samat NA \& Imam SHM, Dengue Disease Mapping with Standardized Morbidity Ratio and Poisson-gamma Model: An Analysis of Dengue Disease in Perak, Malaysia, (8), (2013), pp.46-50.

[3] Kristiani F, Samat, NA \& Bin Ab Ghani S, The SIR-SI model with age-structured human population for dengue disease mapping in Bandung, Indonesia. Model Assisted Statistics and Applications, 12(2), (2017), pp.151-161

[4] Triampo W, Baowan D, Tang IM, Nuttavut N, \& Doungchawee G, A Simple Deterministic Model for the Spread of Leptospirosis in Thailand, (2006), pp.22-26.

[5] Pongsumpun P, Mathematical Model for the Transmission of Lep tospirosis in Juvennile and Adults Humans, (2012), pp.266-271.

[6] Faine S, Guidelines For The Control of Leptospirosis. Melbourne, Australia: WHO Offset Publication (1982), No.67. 